\title{
CD19CAR-CD3zeta-4-1BB-CD28-expressing Autologous T-Lymphocytes
}

National Cancer Institute

\section{Source}

National Cancer Institute. CD19CAR-CD3zeta-4-1BB-CD28-expressing Autologous TLymphocytes. NCI Thesaurus. Code C116069.

Autologous T-lymphocytes transduced with a retroviral vector expressing a chimeric antigen receptor (CAR) consisting of an anti-CD19 scFv (single chain variable frag ment) coupled to three co-stimulatory signaling domains derived from CD28, 4-1BB (CD137), and the zeta chain of the T-cell receptor ( $T C R$ )/CD3 complex (CD3-zeta), with potential immunomodulating and antineoplastic activities. Upon transfusion, the CD19CARCD3zeta-4-1BB-CD28-expressing autologous T-lymphocytes direct the T-lymphocytes to CD19-expressing tumor cells and induce their selective toxicity. CD28, a T-cell surfaceassociated co-stimulatory molecule, is required for T-cell activation, proliferation, and survival. The 4-1BB co-stimulatory molecule signaling domain enhances activation and signaling after recognition of CD19. CD3-zeta is a transmembrane signaling adaptor polypeptide that regulates the assembly of TCR complexes, modulates the expression of the complex on the cell surface and plays a key role in antigen recog nition. CD19 antigen, a B-cell specific cell surface antigen, is expressed in all B-cell lineage malignancies. 